Meta

Journal des traducteurs

Translators' Journal

\title{
Comment rendre en français les termes anglais dérivés et composés à la fois?
}

\section{Charlotte Schapira}

Volume 32, numéro 3, septembre 1987

La fertilisation terminologique dans les langues romanes

URI : https://id.erudit.org/iderudit/004041ar

DOI : https://doi.org/10.7202/004041ar

Aller au sommaire du numéro

Éditeur(s)

Les Presses de l'Université de Montréal

ISSN

0026-0452 (imprimé)

1492-1421 (numérique)

Découvrir la revue

Citer cet article

Schapira, C. (1987). Comment rendre en français les termes anglais dérivés et composés à la fois ? Meta, 32(3), 342-346. https://doi.org/10.7202/004041ar d'utilisation que vous pouvez consulter en ligne. 


\section{COMMENT RENDRE EN FRANÇAIS LES TERMES ANGLAIS DÉRIVÉS ET COMPOSÉS À LA FOIS?}

CHARLOTTE SCHAPIRA

Israeli Institute of Technologie, Haïfa, Israël

La structure anglo-américaine $\mathrm{N}_{1} \mathrm{~N}_{2}$ est construite à partir d'un élément de base $\mathrm{N}_{2}$ - dérivé verbal à suffixe d'agent ou d'instrument (-er, plus rarement -or) : cutter, grader, layer, loader, unloader, etc.

Les composés désignent des dispositifs, des machines ou des parties de machines destinés à une opération que subit $\mathrm{N}_{1}$ :

oven unloader, pipe layer, plug cutter, etc.

La transposition de ces termes en langues romanes pose un problème réel, que reflètent les listes de néologismes spontanés et planifiés. L'examen de ceux-ci révèle d'ailleurs une complication supplémentaire : la difficulté ne se présente, en effet, que pour les termes composés, le dérivé de base $\left(\mathrm{N}_{2}\right)$ trouvant aisément un équivalent français en -eur ou -euse. Les exemples de vocables anglo-américains désignant machines, instruments, outils et dispositifs en -er/-or transposés en -eur/-euse sont si nombreux, par exemple, dans les derniers numéros du Journal officiel de la langue française et dans les différents vocabulaires spécialisés français et canadiens, que l'on est obligé de constater que ce problème, du moins, est définitivement réglé. En voici seulement quelques exemples : blinker/clignoteur; booster/pousseur, propulseur, suramplificateur ; damper/ amortisseur ; dealer/revendeur ; descrambler/descrambouilleur ; dispatcher/largueur, répartisseur ; dumper/moto basculeur ; freezer/congélateur ; inverter/convertisseur ; liner/inhibiteur; launcher/lanceur et back-filler/remblayeuse; grader/niveleuse; gritspreader/gravillonneuse ; fryer/friteuse ; icer/glaceuse ; labeler/étiqueteuse ; etc.

L'équivalence entre -er/-or et -eur/-euse en français semble s'imposer de soi et représente une solution heureuse à tous points de vue. Pour commencer, -or est d'origine latine (cf. The Concise Oxford Dictionary) et il est arrivé en anglais par le biais, précisément, du français -eur, dont il a gardé la principale signification : agent, instrument, machine. Ceci permet parfois de garder en français le même radical qu'en anglais, ce qui de l'avis de D. Bécherel (1981 : 129) accroît les chances d'intégration dans la langue du néologisme : container/conteneur; scanner/scanneur; processor/processeur, etc. Et, même avec un radical français différent, le dérivé français s'apparente assez par sa sonorité à son équivalent anglais pour ne pas gêner l'usager. Les désavantages sont minimes, mais ils méritent peut-être d'être pris en considération : nombre de nouvelles créations (très rares, il est vrai) ne passent pas par un verbe. En effet, si scanneur est étayé par l'existence, en parallèle, du verbe scanner (to scan), tel n'est pas le cas pour processeur, séquenceur ou scrapeur, dont les verbes correspondants n'existent pas en français (*processer, *séquencer, *scraper). Bien que le danger ne soit pas imminent, abuser de ce genre d'emprunt pourrait mener à l'affaiblissement d'un des procédés de dérivation les plus motivés du français. 
Quoi qu'il en soit, l'équivalent -er, -or/-eur, -euse étant un fait établi, les composés anglais à partir d'un dérivé en -er/-or tendent, logiquement, à trouver en français des correspondants à un élément de base en -eur/-euse. Ceci a favorisé la création d'un grand nombre de composés syntagmatiques du type

$$
\mathrm{N}_{2} \text { prép. } \mathrm{N}_{1}
$$

qui, malheureusement, se révèlent souvent des remplaçants très insatisfaisants des termes originels. Dans la plupart des cas, les composés anglais $\mathrm{N}_{1} \mathrm{~N}_{2}$ sont donc rendus en français au moyen des deux noms à ordre inversé reliés par un joncteur prépositionnel. Peuvent être relevées, par ordre de fréquence décroissant, les trois prépositions suivantes :

- de : conditionneur d'air (air conditioner) ; alimenteur d'air ou (air supplier) ; mesureur de fournée ou doseur de fournée (batch measurer) ; chargeur de batterie (battery charger) ; enveloppeuse de boîtes (box wrapper) ; râpeuse - effileuse de chocolat (chocolate shaver); façonneuse de pâte (bread moulder); déchargeur du refroidisseur (cooler unloader), graisseuse de moules (pan greaser) ; simulateur de vol (fight simulator); enregistreur d'accidents (crash recorder); collecteur de données (data collector) ;

- à : cette préposition apparaît rarement comme substitut de de dans les néologismes français (cf. toutefois épaississeur à boue), qui la réservent pour l'expression de la modalité (réacteur à eau bouillante, réacteur à double flux, avion à réaction, avion à flèche variable, brûleur à double débit, etc.). Cependant, elle peut être relevée souvent dans les néologismes canadiens. Ainsi, dans le seul vocabulaire des boulangers et pâtissiers (publié par le gouvernement du Québec, Office de la langue française, 1981), nous avons trouvé les termes suivants où $\mathrm{N}_{1}$ est objet direct du verbe dont dérive $\mathrm{N}_{2}$ : ensacheuse à pains (bread bagger); refroidisseur à pains (bread demoulder, bread depanner); emmouleuse à pain (bread panner); enrobeuse à gâteaux (cake coater) ; améliorant à gâteaux (cake improver) ; tempéreuse à chocolat (chocolate tempering machine).

Bien que les usagers qui exercent la profession ne risquent pas de se méprendre sur l'interprétation correcte de ces composés, l'ambiguité est gênante pour le nonspécialiste. Ainsi, en l'absence des définitions, l'on peut hésiter à comprendre les vocables suivants selon le modèle de la transitivité ou celui de la modalité : band slicer trancheuse à rubans (pour les rubans ou qui fonctionne à l'aide des rubans?); cleated belt conveyor convoyeur à taquets ; air blast freezer congélateur à air forcé, etc. L'on pourrait arguer que cette ambiguïté existe déjà en anglais, mais, précisément, ne serait-il pas utile de résoudre cette difficulté en donnant, en français, la priorité à la compréhension ? (cf. Bécherel $1981: 130$ ) lavoyer).

- en : maintien en position (station keeping); déversement en radar (radar

Ces composés syntagmatiques, qui présentent d'emblée le désavantage de la longueur, sont encore alourdis, parfois, par le fait que, en anglais, $\mathrm{N}_{1}$ comporte un déterminant, ou que ce déterminant est jugé nécessaire en français. Tel est le cas pour le récupérateur de vapeurs d'huile (oil mist collector), pour le réchauffeur de rideau d'air (air curtain heater), pour la façonneuse de pâte à pain (bread moulder), pour la chambre de lavage des paniers (cabinet rack washer), pour le batteur à glace à gâteaux (cake icing beater), ou pour le broyeur de boîtes de conserves (can crucher). Il est évident que ces termes ont peu de chances d'intégration dans la langue. Pour les mêmes raisons, une autre solution proposée par les néologismes inspire le même pessimisme : il s'agit des locutions machine à + verbe (inf) + objet direct : machine à traitement de texte (word processor); machine à couper les biscuits en concurrence avec coupeuse à biscuits et façonneuse à biscuits (cookie cutter); machine à fermer les emballages (package 
closer) ; machine à décorer les gâteux (cake decorator) ; machine à déposer les gâteaux (cake decorator) ; machine à diviser les gâteaux (cake divider); machine à garnir les gâteaux (cake filler) ; machine à changer les sacs (bag changer), machine à fermer les sacs (bag closer), etc.

Ces termes français sont si lourds qu'on ne peut y voir qu'une solution transitoire, en attendant des trouvailles plus heureuses, qui puissent réellement être intégrées dans le langage courant.

À côté de ces procédés plus ou moins fréquents, nous avons relevé dans les vocabulaires spécialisés des créations sporadiques très réussies mais qui, malheureusment, ne sont possibles que dans des cas très spéciaux : star tracker, par exemple, a été adopté sous la forme $\mathbf{N}+$ Adj : suiveur stellaire. Une telle structure, presque idéale pour les termes qui nous occupent, n'a pourtant pas d'avenir parce que très peu de $\mathrm{N}_{1}$ produisent des adjectifs par dérivation suffixale. Celui-ci est d'ailleurs le seul exemple de ce type que nous ayons trouvé. Une autre création isolée est peson pour weight indicator, remarquable par sa concision et par son expressivité. Méritent aussi d'être cités les quelques termes à deux noms juxtaposés, préférables eux aussi aux composés à joncteur prépositionnel non seulement à cause du raccourci, mais aussi parce qu'ils s'inscrivent dans une tendance actuelle plus large du français, qui produit spontanément d'innombrables composés de ce type : pétrisseur-mélangeur (batch-mixer); pulvérisateur-mélangeur (pulvimixer); orienteur-marqueur (path finder); chargeuse-pelleteuse (backhoe loader). Enfin, le Journal officiel ( $\mathrm{n}^{\circ}$ 1468) mentionne aussi l'adoption du terme anglais hydrocracker resté tel quel en français (bien que dans d'autres composés l'élément cracker soit devenu cracqueur).

C'est parmi ces créations sporadiques qu'on aurait dû classer, il y a quelques années à peine, les termes correspondant à la structure verbe + objet direct. Dans un sondage des arrêtés officiels effectué en 1981, D. Bécherel n'en relevait que trois : tire-veine (stripper) ; pose-tube (pipe layer) et tâte-ferraille (funk feeler). Ces dernières années, pourtant, ces créations, encore timides, il est vrai, se sont multipliées de manière significative. On relève dans les arrêtés officiels français et dans les divers vocabulaires spécialisés (cf. références) : porte-annonces (folder) ; pose-tubes (side boom) ; chauffe-caméra (blanket heater) ; lance-câbles (line throwing gun), pour ne plus parler du célèbre remue-méninges. On relève aussi, sur le même modèle tire-bras, guide-bande, guide-mains, guide-câbles, pare-grûmes, porte-grappin, tourne-billes. L'on a répertorié dans le seul vocabulaire de l'équipement de protection individuelle (Néologie en marche, $\mathrm{n}^{\circ} 22,1980$ ) jusqu'à douze composés protège + nom (protège-main, protège-paume, protège-nuque, protège-pied, protège-tibia, etc.) et, ainsi que le note le $G L L F$, ces noms constituent une série ouverte. Les dictionnaires et les vocabulaires spécialisés n'en citent que les termes lexicalisés, usuels, parfaitement intégrés.

Dans l'équipement de voiture ce type de noms est aussi très fréquent : allumecigare, compte-tours, repose-tête, repose-bras, divers composés du verbe lever (lèveglaces, lève-vitres).

Les composés s'emploient couramment en apposition à un support nominal : commande de lève-bras, coquille porte-cellule, panneau porte-annonces, filtre passe-tout, frégate lance-engins, disque porte-têtes, prise tourne-disque (technique de radio), etc.

Dans les vocabulaires canadiens, l'équivalence de ces termes avec la structure anglaise $\mathrm{M}_{1} \mathrm{~N}_{2}$ est évidente; ainsi, nous relevons :

- dans le vocabulaire de la mécanique forestière : attrape-chaîne (chain catcher), pare-billes (log bumper), montant pare-branches (limb riser) à côté de créations locales comme guide-chaîne, porte-outil, écran pare-broussailles ; 
- dans le vocabulaire des boulangers-pâtissiers : lave-moules (baking pan cleaner ou pan washer), lève-cuve (bow lift), chariot porte-moules (bread pan dolly), ouvreboîte (can opener et can perforator), lave-convoyeur (conveyor washer), ramasse-miettes (crumb collecting tray), lève-pétrin (dough through elevator), monte-charge (elevator), coupe-pâte (scraper) ;

- dans le vocabulaire de la perceuse électrique et ses outils coupants : taillebouchon (plug cutter).

Ce moyen de composition lexicale gagne incontestablement du terrain en ce qui concerne les néologismes. Cela n'est nullement étonnant si l'on prend en considération l'énorme production spontanée de composés sur ce modèle, composés qui, eux aussi, désignent des dispositifs, objets ménagers de toutes sortes, instruments de laboratoire et de cuisine, et même différents gadgets. Citons seulement attache-caravane, attacheremorque, chauffe-biberons, essuie-tout, monte-camions, monte-meubles, montemalades, mouche-bébé, mange-disques, pèse-personne, pèse-bébé, porte-bloc, portepeigne et brosse, porte-tout, ramasse-couvert, protège-documents, protège-matelas, protège-slip, etc. Cette production est même si importante que l'on peut se demander pourquoi elle n'a pas inspiré davantage de néologismes planifiés. Pourtant, cette solution à peine esquissée pour la transposition en français de la structure $\mathrm{N}_{1} \mathrm{~N}_{2}$ semble de loin supérieure à celles que nous avons déjà énumérées, non seulement parce qu'elle va dans le sens de la production française spontanée, mais aussi - et surtout - parce qu'elle constitue une structure commune à toutes les langues romanes. En effet, de nombreux ouvrages (depuis Darmesteter en 1874 à nos jours, en passant par les études de Marouzeau, jusqu'à plusieurs articles très récents) examinent ce procédé de formation lexicale des points de vue les plus divers. Il a déjà été démontré qu'il s'agit là d'un phénomène qui remonte au latin médiéval, connu dans toutes les langues romanes et attesté en français dès le XII et le XIII ${ }^{e}$ siècles (crève-coeur, abat-vent, couvre-feu). Les créations de ce type ont abondé à toutes les époques de la langue, celles que nous avons gardées ne représentant qu'une partie de la production totale. Le thème verbal confêre une grande expressivité aux composés. On peut diviser ceux-ci, en gros, en deux catégories distinctes, selon chacun des deux critères suivants : animés (porte-parole)/objets (portefeuille) ; péjoratifs (fainéant, tord-boyaux)/non-péjoratifs (garde-côte, garde-manger) (cf. à ce propos Darmesteter, 1934 et Marouzeau, 1952a).

Parmi les langues romanes, c'est l'italien qui a connu et connaît encore la production la plus importante de ces noms dans toutes les catégories citées. Le français semble privilégier aujourd'hui les non-animés, en particulier ceux qui constituent l'objet de notre étude. L'affinité des composés romans verbe + nom avec les anglo-américains $\mathrm{N}_{1} \mathrm{~N}_{2}$ est basée, avant tout, sur la syntaxe sous-jacente de ces vocables que Darmesteter appelle les " composés-phrases". En français le nom est, dans la plupart des cas, objet direct du verbe qui le précède ; en anglais $\mathrm{N}_{1}$ est l'objet direct du verbe dont dérive $\mathbf{N}_{2}$. Cette équivalence a été définitivement démontrée à la fin du XIX ${ }^{\mathrm{e}}$ siècle, quant le néologisme américain sky scraper a été uniformément rendu dans toutes les langues européennes par gratte-ciel (en langues romanes) et gratte-nuages (en langues germaniques et en roumain). Mais cette correspondance n'est pas nouvelle et elle a été, par le passé, la source d'un jeu d'influences constant entre le français et l'anglais : si brise-glace est un rnot du XVIII' équivalent de l'anglais ice-breaker, le chasse-marée, lui, est bien français et remonte au XVIII' siècle.

Concis, expressif et très motivé dans l'esprit de l'usager, ce modèle présente le précieux avantage de permettre la création de termes similaires dans toutes les langues romanes. Parmi celles-ci une seule - le roumain - élève, a priori, un obstacle à ce programme. En effet, bien que le roumain connaisse aussi ce procédé de composition, la 
production locale - très restreinte en comparaison avec celle des autres langues romanes - se limite aux seuls mots animés et péjoratifs, tels que zgîrie-brînză et linge-blide (qui signifient tous deux " avare "), pierde-vară et tîrîie-brîu (fainéant), gură-cască (niais et fainéant), etc. Cette langue semble même refuser énergiquement les non-animés sur ce modèle, puisqu'un grand nombre de noms français désignant dispositifs, instruments et machines ont été importés tels quels du français avec des adaptations minimes concernant la prononciation et parfois le genre. Ces vocables (portavion, portvisit, portt, igaret, portjartier, portmoneur, parbriz, paravan, garderobă, paspoil, tirbus, son, abajur, etc.) sont interprétés en roumain tout au plus comme des dérivés au moyen d'un préfixe étranger (portofel < (fr) portefeuille ; pa,saport < (it) passaporto). Sans favoriser la production locale par analogie (seul parazăpadă est une création originale du roumain, sans correspondant en français), ces emprunts restent cependant une série ouverte aux néologismes français de ce type. Ciobanu et Hasan (1970) relevaient même, il y a vingt ans environ, deux mots qui, pour être restés exceptionnels, n'en refiètent pas moins le besoin que ressent le roumain de s'aligner sur les autres langues romanes dans la voie déjà ouverte par zgîrie-nori (gratte-ciel) : il s'agit de apără-roată et trece-bandă, provenant probablement du français (protège-roue et passe-bande). Bien que ces calques aillent à l'encontre de la tendance naturelle de la langue, le roumain possède assez de ressources pour suivre les autres langues romanes dans une tentative de transposition unitaire de termes anglais $\mathrm{N}_{1} \mathrm{~N}_{2}$.

Les composés à thème verbal représentent donc une solution digne d'intérêt pour les cas où les autres moyens s'avèrent inappropriés ou impossibles. Malheureusement, en français du moins, ces termes sont souvent ressentis comme populaires, leur prestige n'égalant pas celui des dérivés en -eur. Par ailleurs, les néologismes de ce type trahissent une grande répugnance à forger des mots à partir de verbes qui n'ont pas encore produit de composés. La tendance générale est de favoriser les verbes les plus productifs dans le passé (porter, poser, laver, lever) et même de donner une nouvelle acception à des mots déjà existants : ramasse-miettes, chauffe-eau, ouvre-boîte, etc.

Mais cette évolution est déjà bien entamée et elle offre des possibilités que l'on ne manquera certainement pas d'exploiter à l'avenir.

RÉFÉRENCES

BÉCHEREL, Danièle (1981) : "À propos des solutions de remplacement des anglicismes ", la Linguistigue, vol. 17, $\mathrm{n}^{\circ} 2$, pp. 119-131.

CIOBANU, Fulvia et Finuta HASAN (1970) : Formarea cuvintelor in limba românaă, vol. 1 : Compunerea, Academia Republicii Socialiste România.

DARMESTETER, Arsène (1934) : Cours de grammaire historique de la langue française, édité par L. Soudre, $11^{\mathrm{e}}$ éd., vol. 3, pp. 48-51, \$301.

DARMESTETER, Arsène (1967) : Traité de la formation des mots composés dans la langue française, Paris, $2^{\mathrm{e}}$ éd., revue, corrigée et en partie refondue par Gaston Paris, pp. 168-234.

MAROUZEAU, Jean (1952a) : "Composés à thème verbal ", in le Français moderne, vol. 20, 2, pp. 81-86.

MAROUZEAU, Jean (1952b) : "Thèmes verbaux en français ", in le Français moderne, vol. 20, 2, pp. 161-164.

SCHAPIRA, Charlotte (1982) : "Les noms composés verbe + objet direct", in Travaux de linguistique et de littérature, vol. 20,1 , pp. 271-282.

SCHAPIRA, Charlotte (1985) : "Les composés roumains à thème verbal et leur place dans l'ensemble des langues romanes ", in Revue de linguistique romane, vol. 49, 193-194, pp. 15-26.

Les néologismes cités ont été relevés dans les revues suivantes :

L'Actualité terminologique (1984) : $17, \mathrm{n}^{\circ} 3$.

Datations et documents lexicographiques, $2^{\mathrm{e}}$ série (1984) : nos 24 et 25.

Défense de la langue française (1984) : $\mathrm{n}^{\circ} 121$.

Enrichissement du vocabulaire (1985) : publication du Journal officiel, $\mathrm{n}^{\circ} 1468$

Médias et langage (1983) : $\mathrm{n}^{\circ} 17$.

Néologie en marche $(1980): n^{\circ} 22(1982): n^{\circ} 33(1983): n^{\circ} 34$ et 35 .

La Revue du traducteur (1983) : nos 27 et 28 . 